\title{
Synchronization Rates in Classes of Relaxation Oscillators
}

\author{
Shannon R. Campbell, DeLiang Wang, Fellow, IEEE, and Ciriyam Jayaprakash
}

\begin{abstract}
Relaxation oscillators arise frequently in physics, electronics, mathematics, and biology. Their mathematical definitions possess a high degree of flexibility in the sense that through appropriate parameter choices relaxation oscillators can be made to exhibit qualitatively different kinds of oscillations. We study numerically four different classes of relaxation oscillators through their synchronization rates in one-dimensional chains with a Heaviside step function interaction and obtain the following results. Relaxation oscillators in the sinusoidal and relaxation regime both exhibit an average time to synchrony, $\left\langle T_{S}\right\rangle \sim n$, where $n$ is the chain length. Relaxation oscillators in the singular limit exhibit $\left\langle T_{S}\right\rangle \sim n^{p}$, where $p$ is a numerically obtained value less than 0.5 . Relaxation oscillators in the singular limit with parameters modified so that they resemble spike oscillations exhibit $\left\langle T_{S}\right\rangle \sim \log (n)$ in chains and $\left\langle T_{S}\right\rangle \sim \log (L)$ in two-dimensional square networks of length $L$. Finally, using a sigmoid interaction results in $\left\langle T_{S}\right\rangle \sim n^{2}$, for relaxation oscillators in the sinusoidal and relaxation regimes, indicating that the form of the coupling is a controlling factor in the synchronization rate.
\end{abstract}

Index Terms-Coupled oscillators, neural dynamics, relaxation oscillators, synchronization rate, synchrony.

\section{INTRODUCTION}

$\mathbf{T}$ HE STUDY of coupled oscillators reveals a rich diversity of behaviors and has many ties to physical, chemical, and biological phenomena [24], [49]. The study of how coupled oscillators achieve synchrony is important because of its relevance to experimental observations of synchronous neural firing patterns of various mammalian, insect, and reptilian species [27], [33], [35], [38].

The phrase "relaxation oscillations" was coined by Van der Pol in 1926 in his analysis of a triode circuit [44]. A relaxation oscillation is described by two distinct time scales: a slow time scale, which originally reflected the charging of a capacitor, and a fast time scale, which described a quick discharge. In the equations that describe a relaxation oscillator, the two time scales are typically based on a single parameter $\varepsilon$. As $\varepsilon \rightarrow 0$, the time scales become more disparate.

Relaxation oscillations were immediately recognized as having similarities to biological oscillations. Van der Pol and

Manuscript received June 13, 2003; revised January 16, 2004. This work was supported in part by National Science Foundation Grant IIS-0081058 and in part by the Air Force Office of Scientific Research Grant F49620-01-1-0027.

S. R. Campbell is with the Clinical Center, Diagnostic Radiology Department, National Institutes of Health, Bethesda, MD 20892 USA (e-mail: SCampbell@cc.nih.gov).

D. Wang is with the Department of Computer and Information Science and Center for Cognitive Science, The Ohio State University, Columbus, OH 43210 USA.

C. Jayaprakash is with the Department of Physics, The Ohio State University, Columbus, OH 43210 USA.

Digital Object Identifier 10.1109/TNN.2004.833134 van der Mark may have been the first to use relaxation oscillations to model biological phenomena, the heart beat [45]. In 1952, Hodgkin and Huxley gave a mathematical model of the membrane potential and ionic conductances of a nerve cell using a four variable system of differential equations [19] and these were later simplified to a two variable system of equations that is essentially a relaxation oscillator [16], [32]. Later, Mayeri [28] derived the Van der Pol relaxation oscillator as a quantitative description of his experiments with the cardiac ganglion cells of the lobster. Morris and Lecar [30] also derived a two variable relaxation oscillator in their study of the conductances and currents in the barnacle giant muscle fiber. Due to these direct links to physiology, relaxation oscillators have been frequently used as models of biological behavior [17], [34], [39], [43], [47].

Synchrony in networks of locally coupled relaxation oscillators has been studied previously. Somers and Kopell [39] noted that synchrony occurs more rapidly in relaxation oscillator chains as $\epsilon$ decreases. They explained this quick synchronization for small $\epsilon$ using "fast threshold modulation" and conjectured that the average time to synchrony $\left\langle T_{S}\right\rangle$ increases linearly with $n$, the number of oscillators in a chain. However, it is not known whether this scaling relation is because the oscillations are of relaxation type, or because of the particular interaction used. We present numerical evidence indicating that the linear scaling relation persists when using a Heaviside coupling even when the relaxation oscillators are clearly in the sinusoidal regime, and that relaxation oscillators in the sinusoidal and relaxation regime coupled with a smoothly varying sigmoid function exhibit $\left\langle T_{S}\right\rangle \sim n^{2}$, indicating that the form of the interaction is a critical factor in the synchronization rate. This complements Daido's work [10], indicating that a discontinuous interaction leads to partial perfect synchrony in globally coupled phase oscillator networks with heterogeneous frequencies, and is consistent with simulations and statements made by Somers and Kopell [40] indicating that increasing the steepness of the sigmoid coupling function aids synchronization.

Terman and Wang [43] proved that networks of locally coupled identical relaxation oscillators with a Heaviside type interaction, and as $\varepsilon \rightarrow 0$, can achieve synchrony at an exponential rate independent of the number of oscillators or the dimension of the network. However, this rate of synchronization is possible only when the oscillators are initially on a specific portion of the limit cycle. We study the case in which the initial conditions of the oscillators are randomly and uniformly distributed in a box that bounds the limit cycle and observe different results, i.e., $\left\langle T_{S}\right\rangle \sim n^{p}$ and the observed values of $p$ are less than 0.5 . 
Campbell et al. [7] gave heuristic arguments and numerical evidence that chains of identical spike oscillators achieve synchrony at times proportional to the logarithm of the system size. They also presented simulations indicating that $\left\langle T_{S}\right\rangle \sim \log (L)$ for locally coupled two-dimensional (2-D) square networks of length $L$. We examine relaxation oscillators whose parameters have been adjusted so that they behave qualitatively as spike oscillators and find similar synchronization rates in both 1-D and 2-D networks.

A relaxation oscillator is highly flexible and its parameters can be adjusted so that the type of oscillation it exhibits smoothly varies from sinusoidal to spike oscillations. We briefly define four different classes of oscillations that relaxation oscillators can manifest. For $\varepsilon \sim 1$ relaxation oscillators exhibit sinusoidal oscillations; they can have an almost circular limit cycle and a sinusoidal waveform. We refer to this case as a relaxation oscillator in the sinusoidal regime. As $\varepsilon$ decreases, the waveform takes on a square-wave appearance. The oscillator spends most of its period on the two branches of the $x$ nullcline and quickly switches from one branch to another. These are relaxation oscillations and we refer to oscillators in this parameter regime simply as relaxation oscillators in the relaxation regime. The third type of oscillation occurs with $\varepsilon=0$ and the time spent switching between the two branches is zero. We refer to this as a relaxation oscillator "in the singular limit" [5], [25]. The fourth type of oscillation is also in the singular limit, but with parameters modified so that the oscillator spends a vanishingly small portion of its period on the right branch. We refer to this as a relaxation oscillator in the spiking regime.

While these four classes of oscillations have been created based on the intuition that they have meaningful distinctions, to our knowledge, no objective measure of difference has previously been applied. In this paper, we examine how the average time to synchrony varies with chain length and find three different synchronization rates.

The form of the coupling is also an important factor in the synchronization rate. We explore two different types of coupling, the Heaviside step function and a smooth sigmoid, and find that they yield two different synchronization rates $\left\langle T_{S}\right\rangle \sim n$ and $\left\langle T_{S}\right\rangle \sim n^{2}$, respectively, for relaxation oscillators in the sinusoidal and relaxation regime.

This paper is organized as follows. In Section II, we detail how to modify the parameters of the defining equations of a Terman-Wang oscillator [43] to achieve the four different classes of relaxation oscillators discussed above. In Section III, we describe some basic properties of pairs of relaxation oscillators as well as some properties of relaxation oscillators in the singular limit. In Section IV, we discuss chains of relaxation oscillators. In Section V, we present results for the rate of synchronization in the spiking regime. In Section VI, we compare spike oscillators with relaxation oscillators in the spiking regime. In Section VII, we show our data for the synchronization rate in the singular limit. In Section VIII, we present results for the synchronization rates in the relaxation and sinusoidal regime with Heaviside and sigmoid coupling. In Section IX, we give results in 2-D networks in the singular limit and in the spiking regime. Discussions are given in Section X.
$\mathbf{A}$

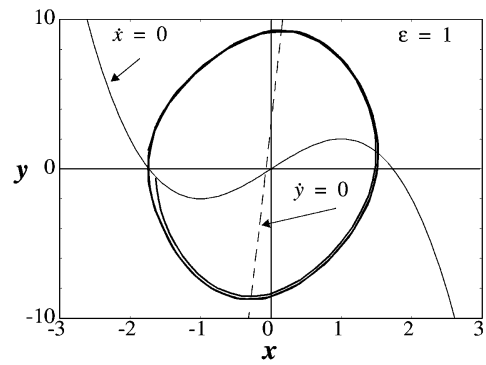

B
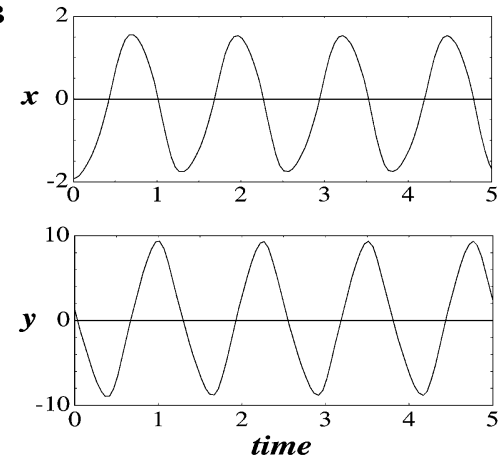

Fig. 1. (a) Limit cycle (thick curve) for system (1) with $\varepsilon=1.0$. The $x$ - and $y$ nullclines are the thinner solid and dashed curves, respectively. (b) Temporal evolution of the $x$ variable and $y$ variable. The parameters are $\lambda=3, \gamma=42$, and $\beta=10$.

\section{DifFERENT Classes OF RELAXATION OSCILlATORS}

We examine a Terman-Wang relaxation oscillator [43] which is simplified from the Morris-Lecar model of neural behavior [30]

$$
\begin{aligned}
& \dot{x}=3 x-x^{3}-y \\
& \dot{y}=\varepsilon(\lambda+\gamma \tanh (\beta x)-y) .
\end{aligned}
$$

Fig. 1(a) displays the limit cycle for (1), using $\varepsilon=1$, and it does appear qualitatively sinusoidal, as does the temporal evolution of both variables in Fig. 1(b). As $\varepsilon$ decreases, the system changes smoothly from sinusoidal oscillations to relaxation oscillations. In Fig. 2(a)-(c), we display limit cycles and temporal evolution of the variables for progressively smaller values of $\varepsilon(\varepsilon=0.33$, $\varepsilon=0.1, \varepsilon=0.01$ ). As $\varepsilon$ becomes smaller, the limit cycle moves closer to the cubic $x$ nullcline, $\dot{x}_{j}=0$. An oscillator travels along one branch of the cubic until it reaches the knee (a local extremum) at which point its velocity is dominated by motion along the $x$ direction and it quickly "jumps" to the other branch of the cubic, where it resumes its relatively slower travel along that branch.

We now describe how trajectories for coupled relaxation oscillators are analytically computed in the singular limit, $\varepsilon=0$. In this regime, the motion of an oscillator is determined by a single variable and knowledge of which branch the oscillator is on (see [43]). The fast system of (1) is obtained by setting $\varepsilon=0$, which results in

$$
\begin{aligned}
& \dot{x}=3 x-x^{3}-y \\
& \dot{y}=0 .
\end{aligned}
$$



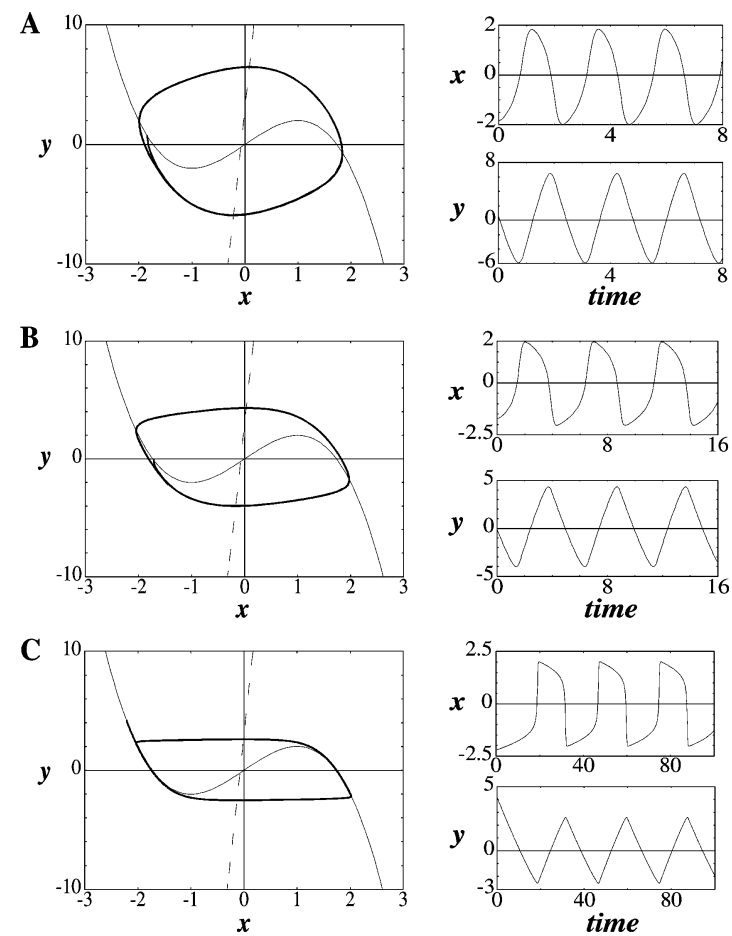

Fig. 2. Three different limit cycles for system (1) and the temporal plots of their $x$ - and $y$-variables are shown for three different values of $\varepsilon$. (a) $\varepsilon=0.33$ (b) $\varepsilon=0.1$. (c) $\varepsilon=0.01$. The $x$ - and $y$ nullclines are the thinner solid and dashed curves, respectively. The parameters are $\lambda=3, \gamma=42$, and $\beta=10$.

The slow system for (1) is derived by introducing a slow time scale $t^{\prime}=\varepsilon t$ and then setting $\varepsilon=0$. The evolution of an oscillator on the left branch in the slow time scale is

$$
\begin{aligned}
& x=h(y) \\
& \dot{y}=\lambda+\gamma \tanh [\beta h(y)]-y
\end{aligned}
$$

where $x=h(y)$ describes the left branch of (1). Because $\beta \gg 1$ and $h(y) \leq-1$, we approximate (3b) with

$$
\dot{y}=\lambda-\gamma-y
$$

For the right branch, these same steps result in

$$
\dot{y}=\lambda+\gamma-y
$$

The set of (2)-(5) describe a relaxation oscillator in the singular limit.

The equations describing $y(t)$ along the left and right branches in the slow time scale are readily solved analytically and many interesting quantities can be computed. For example, we compute the total period of oscillation, $\tau$, the time needed to traverse both branches. The time it takes to travel along the right branch $\tau_{R B}$ from the $y$ position of the left knee $\left(L K_{y}\right)$ to the $y$ position of the right knee $\left(R K_{y}\right)$ is given by

$$
\tau_{R B}=\log \left(\frac{L K_{y}-\gamma-\lambda}{R K_{y}-\gamma-\lambda}\right)
$$

The time needed to travel along the left branch, $\tau_{L B}$, from $R K_{y}$ to $L K_{y}$, is given by

$$
\tau_{L B}=\log \left(\frac{R K_{y}+\gamma-\lambda}{L K_{y}+\gamma-\lambda}\right)
$$

and $\tau=\tau_{R B}+\tau_{L B}$.

The parameters $\lambda$ and $\gamma$ control the height of the smoothly varying portions of the $y$ nullcline. We fix the values $R K_{y}$ and $L K_{y}$ and use $\alpha, \lambda$, and $\gamma$ to control the velocity of an oscillator on either branch. Through reduction of the time an oscillator spends on the right branch in comparison to the time it spends on the left branch, we can make the relaxation oscillator mimic a spike oscillator.

We define the branch ratio, $B_{r}$, as the time an oscillator spends on the right branch divided by the time it spends on the left branch

$$
B_{r}=\frac{\tau_{R B}}{\tau_{L B}}
$$

$B_{r}$ is an analog to the duty cycle. For small $B_{r}$, the oscillation contains an infinitesimal firing time, or voltage spike. We denote relaxation oscillators with $B_{r} \ll 1$ as in the spiking regime.

\section{PAIRS OF RELAXATION OSCILLATORS}

We describe briefly how pairs of relaxation oscillators synchronize and discuss other known solutions. We use a pair of coupled relaxation oscillators given by

$$
\begin{aligned}
\dot{x}_{1} & =3 x_{1}-x_{1}^{3}-y_{1}+\alpha S\left(x_{2}\right) \\
\dot{y}_{1} & =\varepsilon\left(\lambda+\gamma \tanh \left(\beta x_{1}\right)-y_{1}\right) \\
\dot{x}_{2} & =3 x_{2}-x_{2}^{3}-y_{2}+\alpha S\left(x_{1}\right), \\
\dot{y}_{2} & =\varepsilon\left(\lambda+\gamma \tanh \left(\beta x_{2}\right)-y_{2}\right) \\
S(x) & =[1+\exp (\kappa(\theta-x))]^{-1} .
\end{aligned}
$$

The parameter $\alpha$ is the coupling strength and is positive. The interaction term is a sigmoid, mimicking excitatory chemical synapses. Increasing $\alpha S(x)$ elevates the $x$ nullcline $\dot{x}_{i}=0$. This is a property seen in descriptions of neural behavior [16], [19], [30], [48]. When the parameter $\kappa \gg 1$, the interaction approximates a Heaviside step function. The threshold of the interaction term $\theta$ is placed between the left and right branches of the $x$ nullcline, thus, the interaction term is either on or off depending on whether or not an oscillator is on the right or left branch.

When the leading oscillator jumps up from the left branch to the right branch, it is said to "fire." At this time, the interaction term becomes nonzero and excitation is sent to the second oscillator. If the second oscillator is positioned on the left branch slightly above the left knee, then the excitation will raise the $x$ nullcline of the second oscillator and induce it to fire. As the oscillators travel along the right branch, the Euclidean distance between them decreases. An equivalent dynamic occurs when the oscillators jump down. This cycle repeats and the Euclidean distance between the oscillators decreases exponentially to zero. If the leading oscillator does not induce the second oscillator to jump immediately, then other trajectories occur, many of which also lead to a synchronous solution [39], [43]. 


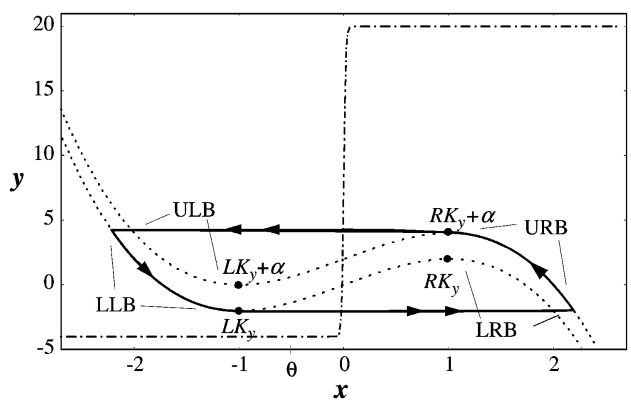

Fig. 3. Plot of the nullclines and the synchronous limit cycle of a pair of relaxation oscillators as defined in (9). The dotted cubics are the excited and unexcited $x$ nullclines, and the dash-dot curve is the $y$ nullcline. The thick solid curve is the synchronous limit cycle for a pair of oscillators and is the result of numerical calculation. The parameters are $\alpha=2, \theta=-0.5, \kappa=5000$, $\lambda=8, \gamma=12, \varepsilon=0.005$, and $\beta=1000$. Upper left branch (ULB), lower left branch (LLB), upper right branch (URB), lower right branch (LRB), $L K_{y}$ is the $y$ position of the left knee, $R K_{y}$ is the $y$ position of the right knee, and $\alpha$ is the coupling strength.

Synchrony can be defined in many ways. In this paper, we use two different measures of synchrony. In the singular limit, the firing time is precisely defined and a network is considered synchronous when the entire chain of oscillators first jumps up or down simultaneously. When $\varepsilon>0$, we sum over the Euclidean distances squared between all pairs of oscillators and divide by the number of pairs to create a measure of the average distance squared between oscillators, $\left\langle D^{2}\right\rangle$. The oscillators are synchronous when $\left\langle D^{2}\right\rangle$ is less than a threshold value. We tested several other measures of synchrony (data not shown) and found no significant difference in our results.

The interaction term alters the limit cycle of the oscillators. When both oscillators jump up from the lower left knee simultaneously, the interaction term raises the $x$ nullclines of both oscillators and they must travel an extra distance $\alpha$ in the $y$ direction. This same additional distance must be traversed when both oscillators jump to the left branch, which is then lowered by an amount $\alpha$. These altered $x$ nullclines are referred to as the upper and lower left (or right) branches. In Fig. 3, we display the synchronous limit cycle of system (9).

The alteration of the limit cycle lengthens the period of the synchronous solution, $\tau_{S}$. The time needed to traverse the upper right branch is

$$
\tau_{U R B}=\log \left(\frac{L K_{y}-\gamma-\lambda}{R K_{y}+\alpha-\gamma-\lambda}\right)
$$

and the time needed to travel from $R K_{y}+\alpha$ to $L K_{y}$, along the lower left branch, is

$$
\tau_{L L B}=\log \left(\frac{R K_{y}+\alpha+\gamma-\lambda}{L K_{y}+\gamma-\lambda}\right) .
$$

The synchronous period is $\tau_{S}=\tau_{U R B}+\tau_{L L B}$, and the branch ratio becomes $B_{r}=\tau_{U R B} / \tau_{L L B}$.

Under certain conditions, a pair of relaxation oscillators can have stable antiphase solutions [23]. In [6], the minimum coupling strength for a pair of coupled Terman-Wang oscillators is derived such that if the coupling strength is below this minimum value, then both antiphase and synchronous solutions occur dependent on the initial conditions. The antiphase solution has a different limit cycle and a different period than the synchronous solution.

We now define the compression ratio $C_{R}$, which is a qualitative measure of how the time difference between two oscillators decreases during a period. The time difference is analogous to a phase difference and is defined when two oscillators are on the same branch. Specifically, $C_{R}$ is the ratio of the initial time difference to the time difference after the two oscillators have jumped up and down together. This ratio is computed using the maximal $y$ distance between the oscillators for an initial jump from the left branch, $\alpha$. While this specific case rarely occurs when using random initial conditions, it is an estimate of the compression per cycle. $C_{R}$ for system (9) is

$$
C_{R}=\frac{\log \left(\frac{c_{6}}{c_{2}}\right)}{\log \left(\frac{c_{8} c_{5}}{c_{1}\left(c_{7}+2 \gamma\right)+2 \alpha \gamma}\right)}
$$

where the values of $c_{i}$ are given by

$$
\begin{array}{ll}
c_{1}=L K_{y}-\lambda-\gamma & c_{5}=L K_{y}+\alpha-\lambda-\gamma \\
c_{2}=L K_{y}-\lambda+\gamma & c_{6}=L K_{y}+\alpha-\lambda+\gamma \\
c_{3}=R H_{y}-\lambda-\gamma & c_{7}=R K_{y}+\alpha-\lambda-\gamma \\
c_{4}=R K_{y}-\lambda+\gamma & c_{8}=R K_{y}+\alpha-\lambda+\gamma
\end{array}
$$

It can be shown that (12) has its maximum value (fastest synchronization) when $B_{r}=1$, i.e., when the amount of time an oscillator spends on the left branch is equal to the amount of time it spends on the right branch.

\section{Chains OF RelaXation OSCILLATORS}

We define a chain of $n$ relaxation oscillators as follows

$$
\begin{aligned}
\dot{x}_{i} & =3 x_{i}-x_{i}^{3}-y_{i}+\sum_{j} J_{i j} S\left(x_{j}\right) \\
\dot{y}_{i} & =\varepsilon\left(\lambda+\gamma \tanh \left(\beta x_{i}\right)-y_{i}\right) \\
S(x) & =[1+\exp (\kappa(\theta-x))]^{-1} .
\end{aligned}
$$

The coupling strengths are normalized using

$$
J_{i j}=\frac{\alpha}{Z_{i}} C_{i j}
$$

matrix $C_{i j}$ represents nearest neighbor coupling

$$
\begin{array}{ll}
C_{i j}=1, & \text { if } j=i \pm 1 \\
C_{i j}=0, & \text { if } j \neq i \pm 1
\end{array}
$$

and $Z_{i}$ is the number of nearest neighbors that oscillator $i$ has. Both $i$ and $j$ vary between 1 and $n . Z_{i}=2$ except for $i=1$ and $i=n$, where $Z_{i}=1$. This normalization ensures that all oscillators have the same trajectory in phase space when synchronous regardless of how many neighbors they have [46].

We briefly describe how to simulate networks of oscillators in the singular limit. In Section III, we showed how the period could be exactly calculated by determining the time spent traveling each branch. Similarly, given an initial position, the time it takes an oscillator to travel to a local extremum of the cubic and jump can be computed analytically [see (4) and (5)]. For 
a system of two or more oscillators, we identify the oscillator that will next jump and at what time it will jump. All oscillators are updated to this time. The interactions between the oscillator that jumped and its neighbors are modified, and this step repeats until no jump occurs. The oscillator to next jump is determined and the above procedure begins again. This algorithm is called the singular limit method, is described in [25], and is used in all our simulations in the singular limit. It allows us to simulate large numbers of oscillators $\left(n=10^{5}\right)$ efficiently. In the nonsingular limit, we use a Runge-Kutta integration method and present data from smaller chain lengths $(n=50)$.

Similar to pairs of relaxation oscillators, in chains of relaxation oscillators, the coupling strength needs to be greater than a specific value, otherwise both desynchronous and synchronous solutions are possible depending on initial conditions. This minimum coupling strength for Terman-Wang oscillator chains is derived in [5]. We focus on the properties of synchronization, and for all results in this paper, we use a coupling strength larger than the minimum coupling strength.

Also, we believe that in one-dimensional (1-D) networks of identical relaxation oscillators (14), traveling waves can only arise if the topology of the network is a ring. Traveling waves in rings are detailed in [5] and basically require that the oscillators have a specific order and specific temporal relationships so that they lead one another around the limit cycle. The time difference between the oscillators is limited by the amount of time spent on the right branch (because at least two or more oscillators must be on the right branch at all times to maintain the traveling wave). As the time spent on the right branch becomes small, a traveling wave must consist of a large number of oscillators evenly positioned on the limit cycle, and thus, the conditions for creating a traveling wave become a small volume of phase space. Also, traveling waves are thought to occur only in ring topologies, in which the ordering can be preserved. At the chain ends, it seems unlikely that the wave is able to reflect, or in other words, reverse the ordering of all oscillators in the chain.

Because the coupling strength used in all simulations presented in this paper is above the limit required for one known type of antiphase solution (described in [5]), and because we doubt that traveling waves can exist in chains, we believe that synchrony is the only solution available for system (15). However, we can not rule out the possibility of other nonsynchronous solutions. In our simulations, we did not observe any other final state of the system besides synchrony.

\section{SynCHRONIZATION IN CHAINS OF RELAXATION OSCILLATORS IN THE SPIKING REGIME}

Fig. 4 displays $\left\langle T_{S}\right\rangle / \tau_{S}$ as a function of $\log _{10}(n)$ for chain lengths that vary from 2 to $10^{5}$, with $B_{r}=8.4 \times 10^{-4}$. Our results are consistent with an average time to synchrony that increases as the logarithm of the system size. This same synchronization rate was observed in simulations of spike oscillator chains with pulsatile coupling [7]. Only several hundred trials are needed to compute the averages because the distribution of the synchronization times does not have a long tail (an example distribution is shown in Fig. 11 and discussed in Section VII).

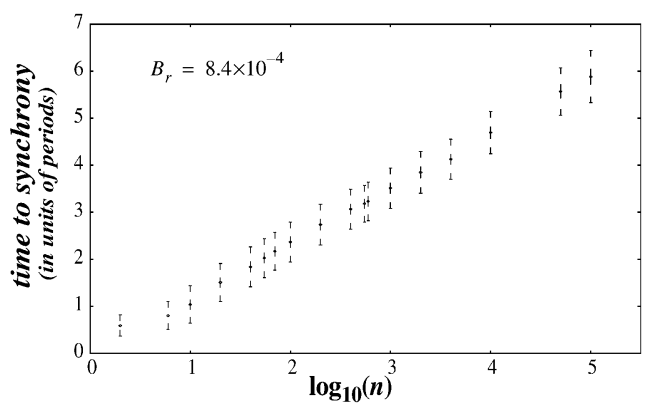

Fig. 4. Average time to synchrony divided by the period of the synchronous solution, $\left\langle T_{S}\right\rangle / \tau_{S}$, as a function of $\log _{10}(n)$ for chains of relaxation oscillators in the spiking regime, $\left(B_{r}=8.4 \times 10^{-4}\right)$. The error bars are shown and indicate the standard deviation. The parameters are $\lambda=2079, \gamma=2082$, and $\alpha=3.5$. the averages are based on 500 trials, except for the last two points representing 50000 and $10^{5}$ oscillators, which are based on 100 and 10 trials, respectively. The initial conditions for each trial were uniformly and randomly distributed in the box that bounds the limit cycle.

However, the data in Fig. 4 could also result from a power-law relationship, $\left\langle T_{S}\right\rangle \sim n^{p}$, with $p \ll 1$, which may be likely considering that relaxation oscillator chains in the singular limit (Section VII) exhibit this scaling relationship. We cannot determine from our numerical results which scaling relationship more accurately describes relaxation oscillators in the spiking regime.

\section{COMPARISON OF RELAXATION OSCILLATORS IN THE SPIKING REGIME WITH SPIKE OSCILLATORS}

\section{A. Comparison of Oscillator Pairs}

Relaxation oscillators are typically thought to have much in common with spike oscillators. Both oscillators are used to model neuronal behavior and both are frequently examined with a discontinuous interaction. We make qualitative comparisons of oscillator dynamics for a pair of Terman-Wang relaxation oscillators and a pair of spike oscillators used in [7].

A network of spike oscillators is defined as

$$
\dot{x}=-x_{i}+I_{0}+\sum_{m, j} J_{i j} \delta\left(t-t_{j}^{m}\right) .
$$

The parameter $I_{0}$ and the threshold control the period of an uncoupled oscillator. The threshold of an oscillator is 1 . When $x_{i}=1$ the oscillator is said to "fire;" its value is instantly reset to 0 and it sends excitation to its neighbors. $t_{j}^{m}$ represents the $m$ firing times of oscillator $j$ and $\delta(t)$ is the Dirac Delta function. The coupling term, $J_{i j}$, is the same as defined in (15). When oscillator $j$ fires at time $t$, oscillator $i$ receives an instantaneous pulse, which increases $x_{i}$ by $J_{i j}$. If $x_{i}$ increases above threshold, then it fires and its value is reset as follows, $x_{i}\left(t^{+}\right)=x_{i}\left(t^{-}\right)+J_{i j}-1$. Since oscillator $i$ fires, immediately oscillator $j$ receives excitation and, thus, $x_{j}\left(t^{+}\right)=J_{j i}$. This particular realization of a network of spike oscillators was called "Model A" in [20].

When one relaxation oscillator fires, it can induce its neighbor to immediately fire, a dynamic that also occurs in spike oscillators. However, in relaxation oscillators, the interaction is of finite duration, unlike the instantaneous interaction between spike oscillators. Further, in relaxation oscillators one oscillator can 
A

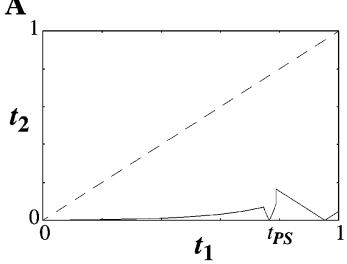

B

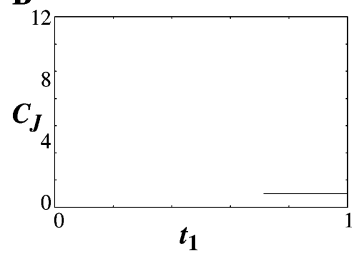

Fig. 5. (a) Return map for a pair of relaxation oscillators. The horizontal axis represents the initial time difference, $t_{1}$, between the oscillators on the lower left branch and the vertical axis represents the time difference between the oscillators when they are next on the lower left branch, $t_{2}$. (b) Plot of the number of periods needed before both oscillators are in the jumping region $C_{J}$, as a function of the initial time difference between the two oscillators $t_{1}$. The parameters are $\lambda=2079, \gamma=2082$, and $\alpha=3.5$.

receive excitation for a short time, which alters the branch the oscillator is on, but when excitation ceases, the position of the oscillator can be identical to what it would have been without excitation, unlike spike oscillators with pulsatile coupling. Also, the interaction increases the synchronous period for relaxation oscillators, while decreasing it for spike oscillators.

We calculate a return map [Fig. 5(a)] for a pair of relaxation oscillators initially placed on the lower left branch. The initial time difference between the two oscillators is represented on the horizontal axis and the vertical axis displays their time difference when they are next on the lower left branch. This return map ignores changes in ordering. We note several features of this return map. The "jumping region" corresponds to those initial conditions in which the firing of one oscillator immediately induces the other to fire. For the parameters used, the jumping region represents the first $70 \%$ of the initial conditions. Just beyond the jumping region, there is an initial time difference, $t_{P S}$, that results in perfect synchrony. Somers and Kopell [39] called the region near this time difference "super compressed," because large initial time differences are mapped into small time differences in just one period. For larger initial time differences, the leading oscillator can jump up and down before the second oscillator can jump up. For the parameters we use, this trajectory also significantly reduces the time difference. In Fig. 5(b), we display the number of periods needed before two oscillators are in the jumping region, $C_{J}$, as a function of their initial time difference. The maximum $C_{J}$ is one.

For a pair of spike oscillators, we display the return map and $C_{J}$ in Fig. 6(a) and (b), respectively, (from [7]). The parameters are such that the jumping regions comprise approximately $70 \%$ of the limit cycle. The spike oscillator pair exhibits an unstable fixed point and many cycles can pass before the oscillators fire synchronously depending on their initial conditions.

\section{B. Comparison of Oscillators Chains}

In Fig. 7, we display $\left\langle T_{S}\right\rangle / \tau_{S}$ as a function of $\log _{10}(n)$ for chain lengths from 5 to $10^{5}$ for spike oscillators and for relaxation oscillators modified so that $B_{r}=0$. The average synchronization time is not noticeably different than with $B_{r}=8.4 \times 10^{-4}$ (data shown in Fig. 4).

We tried to compare oscillators that exhibit the greatest similarity. We chose parameters so that the size of the jumping region is approximately $70 \%$ of the limit cycle for both oscillators. Our
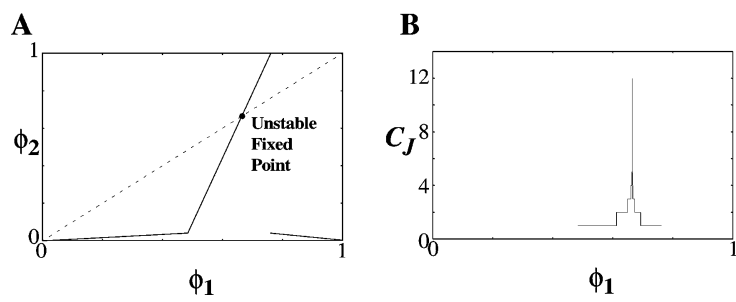

Fig. 6. (a) Return map for two pulse coupled spike oscillators. The phase difference between the oscillators before ( $\phi_{1}$, horizontal axis) and after $\left(\phi_{2}\right.$, vertical axis) they have jumped. (b) Plot of the number of cycles needed, $C_{J}$, before the oscillators are synchronous as a function of $\phi_{1}$. Figure taken from [7].

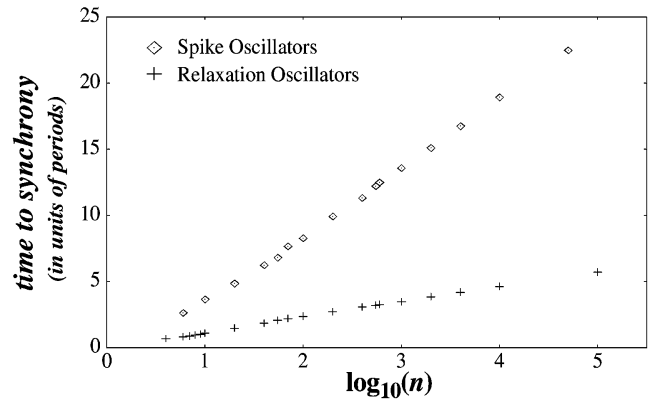

Fig. 7. Plot of $\left\langle T_{S}\right\rangle / \tau_{S}$ as a function of $\log _{10}(n)$ for relaxation oscillator chains (plus signs) with an instantaneous traversal of the right branch and spike oscillator chains (diamonds). Both oscillators have jumping regions which are approximately $70 \%$ of the limit cycle. The data for the spike oscillators is from [7] and the parameters for the relaxation oscillators are $\alpha=3.5, \lambda=4$, and $\gamma=7$. The averages are based on approximately 300 trials except for the point representing a chain length of $10^{5}$ relaxation oscillators, which is based on 25 trials. The initial conditions for the relaxation oscillators were uniformly and randomly distributed on the lower left branch, since it is not possible to place an oscillator on the right branch in this case.

numerical results are consistent with a time to synchrony that increases as the logarithm of the chain length for spike oscillators and relaxation oscillators with $B_{r}=0$. However, relaxation oscillator chains achieve synchrony faster than spike oscillator chains. This difference may be qualitatively explained by the return map for two relaxation oscillators [Fig. 5(a)] and that for two spike oscillators [Fig. 6(a)]. The return map for relaxation oscillators indicates a generally larger compression per period and the number of cycles needed before both oscillators jump together is only one [Fig. 5(b)], while $C_{J}$ for two spike oscillators can be greater than one [Fig. 6(b)]. Based on these return maps, one might expect that the time needed to synchronize a chain of relaxation oscillators would be less than that for a chain of spike oscillators.

In networks of spike oscillators, clusters of synchronous oscillators form and the evolution of the network is such that the number of domain walls does not increase [7]. Assuming that the rate at which clusters merge is a constant that is dependent only on the system parameters, then the number of clusters should decrease exponentially, and this is what is observed numerically [7]. For relaxation oscillators in the spiking regime, we believe that similar dynamics occur. Our preliminary investigations indicate that occasionally, a small number of oscillators switch from one cluster to another, but the formation of new clusters is not observed. If the rate at which clusters merge is a 


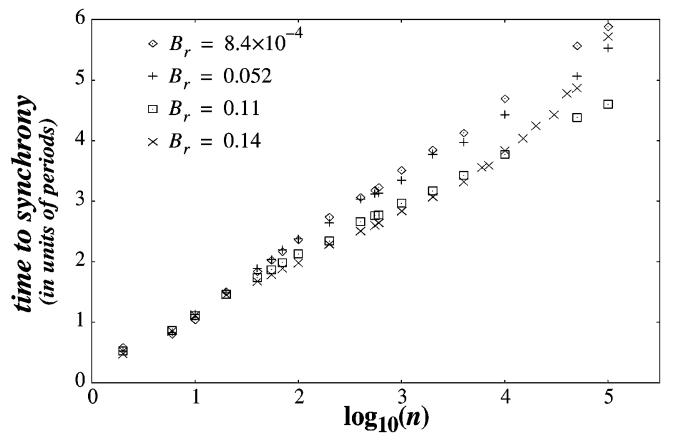

Fig. 8. Plot of $\left\langle T_{S}\right\rangle / \tau_{S}$ as a function of $\log _{10}(n)$ for relaxation oscillator chains in the singular limit. The four different symbols represent four different values of $B_{r}$. The parameters are shown in Table I. The initial conditions were randomly and uniformly distributed within the box bounding the limit cycle. The data for $B_{r}=8.4 \times 10^{-4}$ are described the Fig. 4 caption. For the other three values of $B_{r}$, the averages are based on 250 trials each, except for the data points representing $10^{5}$ oscillators, which are based on 10 trials each.

TABLE I

THE BRANCh RATIOS AND THE PARAMETERS FOR THE DATA DISPLAYED IN FIG. 8

\begin{tabular}{l|c|c|c}
\hline \multicolumn{1}{c|}{$B_{r}$} & $\alpha$ & \multicolumn{1}{c|}{$\lambda$} & $\gamma$ \\
\hline \hline $8.4 \times 10^{-4}$ & 3.5 & 2079 & 2082 \\
\hline 0.052 & 3.5 & 33 & 36 \\
\hline 0.11 & 3.5 & 16 & 19 \\
\hline 0.14 & 3.5 & 12 & 15 \\
\hline
\end{tabular}

constant that depends only on system parameters then we can expect exponential growth of the cluster size.

\section{SYNCHRONIZATION IN CHAINS OF RELAXATION OSCILLATORS IN THE SINGULAR LIMIT}

In Fig. 8, we display $\left\langle T_{S}\right\rangle / \tau_{S}$ as a function of $\log _{10}(n)$ for relaxation oscillators with several different $B_{r}\left(B_{r}=8.4 \times 10^{-4}\right.$, $B_{r}=0.052, B_{r}=0.11$, and $B_{r}=0.14$ ) for chain lengths that vary from 2 to $10^{5}$. The parameters are listed in Table I and were chosen to examine how the average time to synchrony changes as the relaxation oscillators move out of the spiking regime. The data show that chains with $n<20$ have the same average synchronization time. For larger chain lengths, networks with different parameters exhibit different times to synchrony. The diamonds $\left(B_{r}=8.4 \times 10^{-4}\right)$, plus signs $\left(B_{r}=0.052\right)$, and squares $\left(B_{r}=0.11\right)$ all appear to lie on straight lines for values of $n>100$ and are, thus, consistent with $\left\langle T_{S}\right\rangle \sim \log (n)$. The data for $B_{r}=0.14$ (denoted by $\times$ ) are no longer linear on this semilog plot.

The parameters used in Fig. 8 increased the amount of time an oscillator spent on the right branch while attempting to minimize changes in other aspects of the oscillator. Specifically, the distance between the right branch and the $y$ nullcline for $x>0$ decreased while the distance between the left branch and the $y$ nullcline for $x<0$ remained constant. This change decreased the speed of the oscillator on the right branch while not affecting the speed of the oscillator on the left branch. The

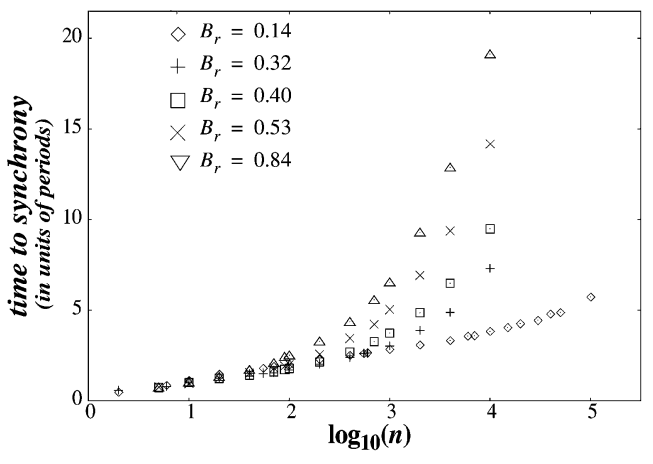

Fig. 9. Plot of $\left\langle T_{S}\right\rangle / \tau_{S}$ as a function of $\log _{10}(n)$ for relaxation oscillator chains in the singular limit. The five different symbols represent relaxation oscillator chains with five different values of $B_{r}$. The averages are based on 250 trials each, except for the data point representing $10^{5}$ oscillators, which is based on 10 trials. The parameters are shown in Table II. The initial conditions were randomly and uniformly distributed within the box bounding the limit cycle.

TABLE II

Branch Ratios and the Parameters for the Data Displayed in Figs. 9 AND 10. THE LAST COLUMN CONTAINS THE SLOPES OF THE LiNES CAlCUlated From the DaTA SHOWN IN FIG. 10

\begin{tabular}{c|c|l|l|l}
\hline$B_{r}$ & $\alpha$ & $\lambda$ & $\gamma$ & slope \\
\hline \hline 0.14 & 3.5 & 12 & 15 & 0.14 \\
\hline 0.32 & 3.5 & 5 & 8 & 0.30 \\
\hline 0.40 & 3.5 & 4 & 7 & 0.33 \\
\hline 0.53 & 3.5 & 3 & 6 & 0.45 \\
\hline 0.84 & 3.5 & 2 & 5 & 0.46 \\
\hline
\end{tabular}

coupling strength was not altered and the position of the synchronous limit cycle remained the same. The period increases by a factor of two as $B_{r}$ goes from zero to one.

The slopes of the lines for $B_{r}=8.4 \times 10^{-4}, B_{r}=0.052$, and $B_{r}=0.11$ decrease as the oscillator spends more time on the right branch, indicating a faster approach to synchrony. This may be qualitatively explained by noting that the compression ratio, $C_{r}$, increases as $B_{r}$ increases.

With $B_{r}=0.14$ the data in Fig. 8 indicate that $\left\langle T_{S}\right\rangle$ no longer increases linearly with $\log _{10}(n)$. This leads us to examine larger values of $B_{r}$. In Fig. 9, we display $\left\langle T_{S}\right\rangle / \tau_{S}$ as a function of $\log _{10}(n)$ with branch ratios larger than those shown in Fig. 8, specifically, $B_{r}=0.14, B_{r}=0.32, B_{r}=0.40, B_{r}=0.53$, and $B_{r}=0.84$; the trend that started in Fig. 8 becomes more pronounced. The parameters are listed in Table II.

In Fig. 10, we display the same data shown in Fig. 9 except that the data are plotted on a log-log graph. Also, we do not show all of the data from Fig. 9 because the average times to synchrony for $n<100$ do not yield straight lines and we assume that they do not reflect the asymptotic behavior of the system. For system sizes from $10^{2}-10^{4}$, our data is consistent with $\left\langle T_{S}\right\rangle \sim n^{p}$. The slopes of the lines were obtained using a least squares fit and are listed in Table II. They vary from 0.46 $\left(B_{r}=0.84\right)$ to $0.14\left(B_{r}=0.14\right)$.

In Fig. 10, the data for $B_{r}=0.14$ is shown in the inset along with error bars representing the addition and subtraction of the standard deviation. The standard deviations for other parameters are not shown, but are similar to those seen in the inset. 


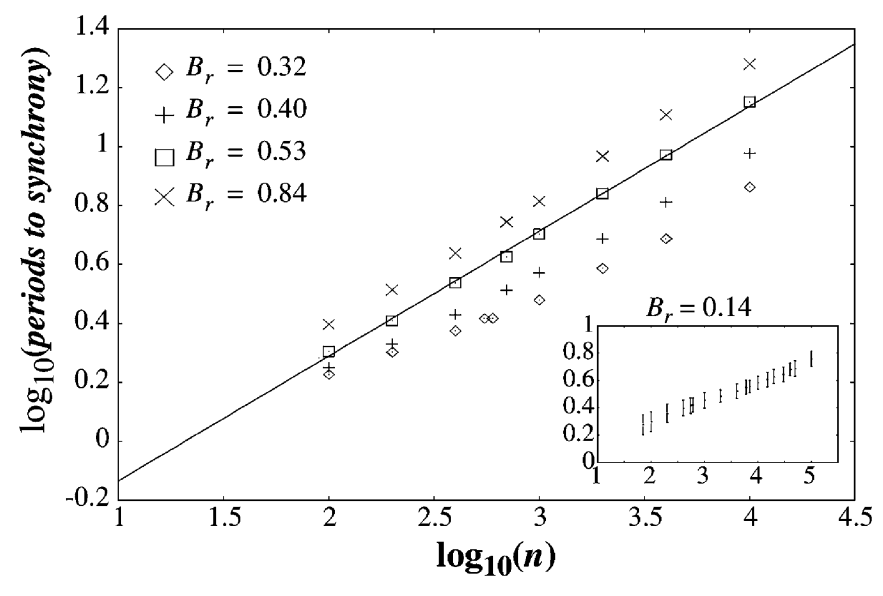

Fig. 10. Log-log plot of $\left\langle T_{S}\right\rangle / \tau_{S}$ as a function of $n$ for the data shown in Fig. 9. The straight line represents a least squares fit to the data for $B_{r}=0.53$. A plot of $\left\langle T_{S}\right\rangle / \tau_{S}$ and its standard deviations for $B_{r}=0.14$ are shown in the inset. The parameters used and the slopes of the lines computed for each value $B_{r}$ of are shown in Table II.

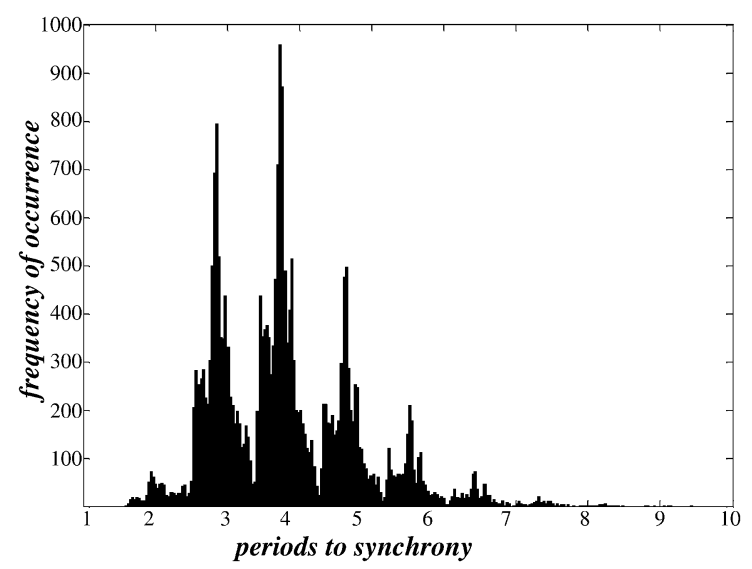

Fig. 11. Histogram of $\left\langle T_{S}\right\rangle / \tau_{S}$ for 25075 trials of a chain of 500 relaxation oscillators in the singular limit, $B_{r}=0.53$, with parameters $\alpha=3.5, \lambda=3$, and $\gamma=6$. The histogram indicates that the distribution of synchronization times does not have a long tail. The initial conditions were randomly and uniformly distributed within the box bounding the limit cycle.

In Fig. 11, we display a histogram of synchronization times for a chain of 500 relaxation oscillators. The histogram indicates that the distribution of synchronization times has the rough appearance of a log-normal distribution if one ignores the periodic structure and examines its envelope. The periodic structure arises because we determine that a system is synchronous when all oscillators jump at the same time. Given random initial conditions, the first oscillator to jump occurs shortly after the start time $(t=0)$ and the synchronization time frequently occurs near an integer interval of the period after that.

The data presented give the impression that $p$ is directly related to $B_{r}$. While it may be true in general that $p$ increases as $B_{r}$ increases, it is not true that $B_{r}$ is the only factor controlling the rate of synchrony. Different parameters can yield identical values for $B_{r}$, but the resultant scaling relation between $\left\langle T_{S}\right\rangle$ and $n$ can appear quite different. Using $\alpha=18, \lambda=49$, and $\gamma=80$, results in an average time to synchrony that increases logarithmically with the system size, even though $B_{r}=0.33$, a value nearly identical to one of the parameter sets shown in
Fig. 10 that exhibits a power law scaling relation. We do not have a detailed understanding of which parameters are related to $p$.

We examined the scaling relation for a variety of coupling strengths and parameters. All parameters tested with $B_{r}<0.1$ exhibited a logarithmic scaling relation, while those with larger values of $B_{r}$ exhibited either logarithmic or power law scaling, with a tendency toward power law scaling.

For system sizes from $10^{2}-10^{4}$, our numerical results are consistent with $\left\langle T_{S}\right\rangle \sim n^{p}$, though we do not know if this relation accurately reflects the asymptotic behavior of the system. This power law scaling is very curious. For systems that exhibit this scaling relation we observe "defects," or spatial arrangements of oscillators resembling traveling waves, arise that hinder the formation of synchrony. These defects have the same frequency as traveling waves and they can remain stable for a short amount of time (several periods) until they merge with a neighboring synchronous cluster. We suspect that they may be the cause of the power law scaling. Because traveling waves occur with decreasing frequency as $B_{r}$ decreases, and because they cannot exist unless the right branch represents a finite portion of the period, it is likely that the logarithmic scaling relation occurs for $B_{r}=0$, and power law scaling for $B_{r}>0$. Unfortunately, we have no theoretical explanations for the power law scaling at this time and refer the reader to [5], which describes traveling waves, specific spin-wave type initial conditions which result in somewhat longer times to synchrony, and other relevant issues.

\section{EFFECTS OF COUPLING FORM ON RELAXATION OSCILLATORS}

We now examine the synchronization rate in chains of relaxation oscillators for $\varepsilon>0$ and with two different couplings, a Heaviside function and a smooth sigmoid.

\section{A. Heaviside Interaction}

In Sections II-VII, relaxation oscillators were in the singular limit and no time was needed for an oscillator to jump from branch to branch. We now investigate the case of $\varepsilon>0$ which results in a finite time needed for an oscillator to jump from branch to branch. This immediately causes a fundamental change in that the time needed for one oscillator to induce another oscillator to jump is finite and information can only propagate from one end of the chain to the other at times proportional to $n$ [39].

In Fig. 12, we present our data for relaxation oscillators in the sinusoidal regime with a Heaviside coupling $(\kappa=5000)$ in the form of histograms of the time to synchrony divided by $n$. Data from two chain lengths $n=25$ and $n=50$, and two values of $\varepsilon(\varepsilon=0.1$ and $\varepsilon=1)$ are shown. Although the histograms are noisy and the variance of the time to synchrony is quite large, the scaled histograms have a qualitative match in their shape and their extent. The values of $\left\langle T_{S}\right\rangle$ and its standard deviation both appear to increase linearly with the system size for both $\varepsilon=0.1$ and $\varepsilon=1$. A value of $\varepsilon=1$ places the relaxation oscillators clearly in the sinusoidal regime and a value of $\varepsilon=0.1$ arguably places the relaxation oscillators in the sinusoidal regime. 
A

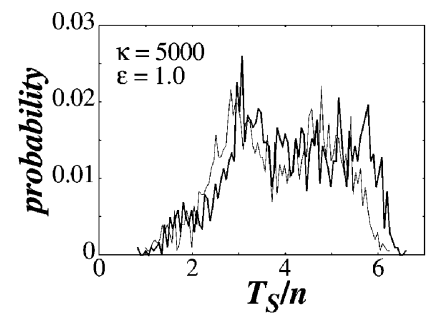

B

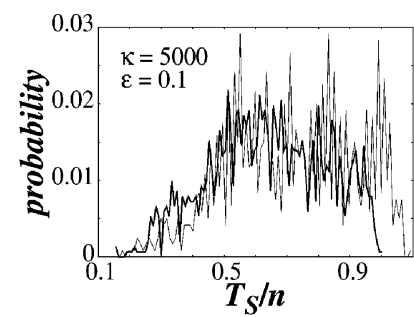

Fig. 12. Histograms of the time to synchrony divided by $n$ using a nearly discontinuous interaction $(\kappa=5000)$ for chains of length $n=25$ (thin) and $n=50$ (thick) (a) Scaled histograms for $\varepsilon=1.0$ are based on 2000 trials for both chain lengths. (b) Scaled histograms for $\varepsilon=0.1$ are based on 1200 and 1500 trials for chain lengths of 25 and 50, respectively. The remaining parameters are $\alpha=6, \lambda=3, \gamma=42, \theta=-0.5$, and $\beta=1000$. Each trial was performed with the initial conditions uniformly and randomly distributed on the lower left branch of the limit cycle.

For $\varepsilon>0$, a network is synchronous when the average Euclidean distance squared between the oscillators $\left\langle D^{2}\right\rangle$ is less than 0.01

$$
\left\langle D^{2}\right\rangle=\frac{2}{n(n-1)} \sum_{i, j<i}^{n}\left[\left(x_{i}-x_{j}\right)^{2}+\left(y_{i}-y_{j}\right)^{2}\right] .
$$

The limit cycle varies in both the $x$ - and $y$-directions by roughly $O(1)$, thus, a threshold of 0.01 for $\left\langle D^{2}\right\rangle$ indicates that the oscillators are relatively close to each other. We tested several measures of synchrony and did not see any discernible difference in our results. An adaptive fifth-order Runge-Kutta method from [36] generated all results for $\varepsilon>0$ (see [5] for more details).

For completeness, we examined pairs of relaxation oscillators for a few parameters with $\varepsilon>1$ and found that there is a transition from synchrony to aperiodicity between $\varepsilon=1$ and $\varepsilon=10$.

\section{B. Smooth Interaction}

In this section, we set $\kappa=1$ and the interaction is a smooth sigmoid instead of an approximate Heaviside step function. Fig. 13(a) displays histograms of the time to synchrony divided by $n^{2}$, for $\varepsilon=1$, and for chains of length $n=25$ and $n=50$. It is evident that this scaling is appropriate. Fig. 13(b) and (c) displays similar histograms with $\varepsilon=0.1$ and $\varepsilon=0.01$ and for these values of $\varepsilon$, the scaled histograms do not line up precisely. Scaling by $n^{2}$ overestimates the time to synchrony by a small but noticeable amount. This is as expected because the interaction is a nontrivial function of the $x$ coordinate and becomes less smooth as $\varepsilon$ decreases.

Our data confirms the numerical results of Somers and Kopell in that relaxation oscillator chains synchronize faster as $\varepsilon$ decreases [39], and in that greater slopes of the interaction term aid synchronization [40]. In Table III, we show a subset of our data for two extreme values of $\kappa$ and several values of $\varepsilon$. Intermediate values of $\kappa$ do not yield readily quantified scaling behaviors, but they are intermediate between $n$ and $n^{2}$. The data are also consistent with a time to synchrony proportional to $\varepsilon^{2 / 3}$. This is in agreement with perturbation analysis for relaxation oscillators, in which an expansion in $\varepsilon$ gives a first term of order $\varepsilon^{2 / 3}$ [2].

Our data may include large errors due to boundary effects that are significant in comparison to the small system sizes $(n=50$

A

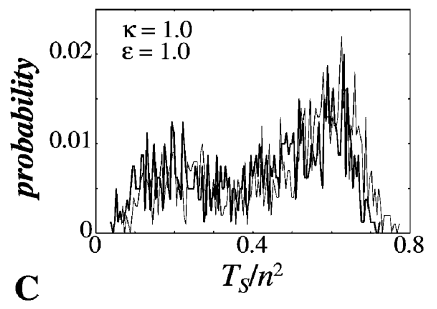

B

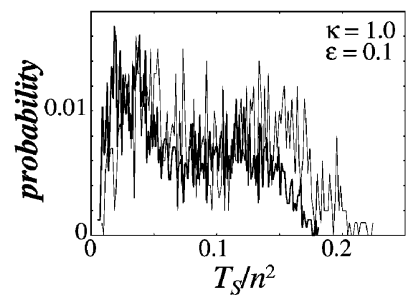

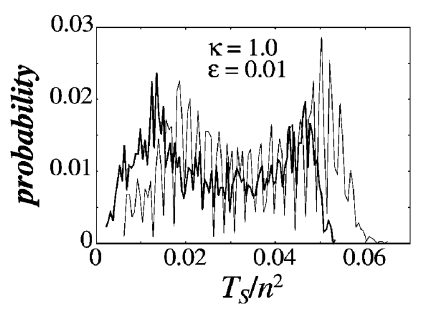

Fig. 13. Histograms of the time to synchrony divided by $n^{2}$ using a smooth interaction ( $\kappa=1$ ) for chains of length $n=25$ (thin) and $n=50$ (thick). (a) The scaled histograms for $\varepsilon=1.0$ are based on 1000 and 800 trials for chain lengths of 25 and 50, respectively. (b) Scaled histograms for $\varepsilon=0.1$ are based on 1000 and 400 trials for chain lengths of 25 and 50, respectively. (c) Scaled histograms for $\varepsilon=0.01$ are based on 4000 and 2500 trials for chain lengths of 25 and 50, respectively. The remaining parameters are $\alpha=6, \lambda=3, \gamma=42$, $\theta=-0.5$, and $\beta=1000$. Each trial was performed with initial conditions randomly and uniformly distributed on the lower left branch of the limit cycle.

TABLE III

The Average Time to Synchrony (IN Periods) For DifFerent VAlues of $n, \varepsilon$, AND $\kappa$. (A) CONTAINS OUR DATA FOR $\kappa=1.0$ AND (B) CONTAINS OUR DATA FOR $\kappa=5000$. THE DATA SUGGEST AN INCREASE IN THE TIME TO SYNCHRONY AS $n^{2}$ FOR $\kappa=1.0$ AND AS $n$ FOR $\kappa=5000$. THE DATA ALSO Suggest That THE Time to SynChrony Is Proportional to $\varepsilon^{2 / 3}$

A

\begin{tabular}{l|l|l|l}
\hline \multicolumn{1}{c|}{$\kappa=1$} & $n=10$ & $n=25$ & $n=50$ \\
\hline \hline$\varepsilon=1.0$ & 61.0 & 292 & 1050 \\
\hline$\varepsilon=0.33$ & 31.8 & 149 & 503 \\
\hline$\varepsilon=0.1$ & 14.7 & 61.5 & 192 \\
\hline
\end{tabular}

B

\begin{tabular}{l|l|l|l}
\hline$\kappa=5000$ & $n=10$ & $n=25$ & $n=50$ \\
\hline \hline$\varepsilon=1.0$ & 35.2 & 96.8 & 179 \\
\hline$\varepsilon=0.33$ & 15.6 & 37.1 & 70 \\
\hline$\varepsilon=0.1$ & 7.76 & 18.0 & 32.5 \\
\hline
\end{tabular}

is the largest), or the effects of correlations that are larger than the size of the system used. In spite of these possibilities, we have good reasons to believe in the scaling relations suggested by our data. One reason is that the histograms for different $n$ have very similar shapes, indicating that these shapes result from the system parameters and not the size of the network. Also, our data indicate an expected proportionality to $\varepsilon^{2 / 3}$, and this proportionality might not be evident if boundary effects are significant. Finally our data are consistent with the results reported in [39], [40], and [46].

It has been shown, that under certain conditions, many forms of oscillators and interactions can be reduced to a system of phase oscillators coupled through a function of their phase differences [11]. To a first-order approximation, this becomes a system of phase oscillators with diffusive coupling, and the time to synchrony is then proportional to the length of the chain squared (more general and complete arguments for this scaling relation are made in [11], [22], [39]). This is a possible explanation for the observed scaling relationship, $\left\langle T_{S}\right\rangle \sim n^{2}$, when the interaction term is a smooth sigmoid. 
A

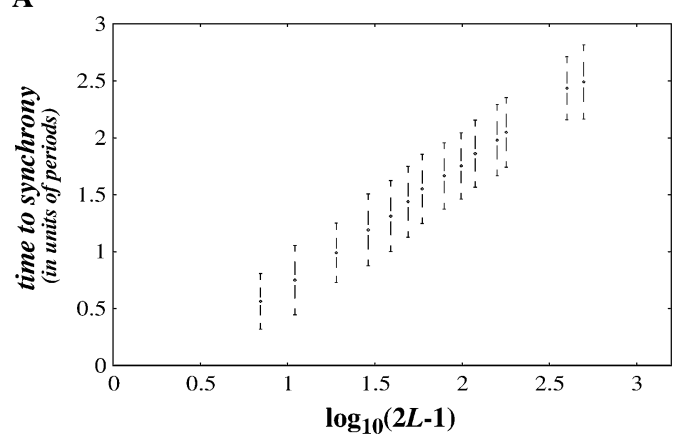

B

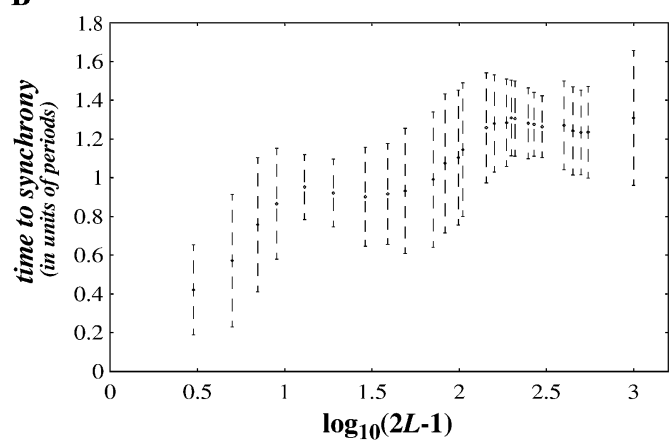

Fig. 14. Plot of $\left\langle T_{S}\right\rangle / \tau_{S}$ as a function as a function of $\log _{10}(2 L-1)$ for $L \times L$ networks of locally coupled relaxation oscillators. All oscillators are coupled with their four nearest neighbors, except those on the borders and the corners, which are connected with three and two neighbors, respectively. The coupling is normalized as described in Section IV. (a) Network parameters are such that $B_{r}=0.021(\alpha=8, \lambda=112$, and $\gamma=115)$, and the averages are based on 300 trials except for the average of the largest square which is based on 100 trials. (b) Network parameters are such that $B_{R}=0.33(\alpha=8, \lambda=8$, and $\gamma=11$ ), and the averages are based on 250 or more trials except for the largest two square sizes, which are based on 150 and 14 trials, respectively. The error bars are shown and indicate the standard deviation. The initial conditions were randomly and uniformly chosen to lie on the lower left branch of the limit cycle to avoid nonsynchronous solutions.

Based on this data, we conjecture that a discontinuous interaction has better properties of synchronization than a smooth interaction for many types of oscillators. This conjecture is in agreement with the work of Daido [10] in which a step-like interaction perfectly synchronizes a portion of the oscillators in a globally coupled network with a normal distribution of intrinsic frequencies, whereas a smooth coupling cannot. Also, chains of pulse coupled spike oscillators synchronize at times proportional to $\log (n)$ [7], but when weakly coupled with a smoothly varying function, the scaling relation changes completely [22]. These data indicate that a discontinuous interaction may be generically better at producing quick synchronization than a smooth interaction.

\section{SYNCHRONIZATION IN 2-D NETWORKS OF RELAXATION OSCILLATORS IN THE SINGULAR LIMIT}

Because synchrony was quickly attained in chains of oscillators, we examined 2-D locally coupled networks also. Here we only examined relaxation oscillators in the singular limit and in the spiking regime because they can be numerically integrated rapidly [25] enabling us to present data for large network sizes.

In Fig. 14(a), we display $\left\langle T_{S}\right\rangle / \tau_{S}$ as a function of $\log _{10}(2 L-1)$ for square networks of length $L$ varying from 4

to 250 , with $B_{r}=0.021$. Our data are consistent with $\left\langle T_{S}\right\rangle \sim$ $\log (L)$. This scaling relation is the same as that observed in 2-D networks of spike oscillators [7], thus, again indicating that relaxation oscillators in the spiking regime appear to be fundamentally similar to spike oscillators.

In rings of oscillators, traveling waves can occur. In 2-D networks with periodic boundary conditions, traveling waves and other more complex patterns occur. Rotating waves were observed but only when initial conditions were randomly distributed within the box bounding the limit cycle (see [5] for more details). When we restricted the initial conditions to the lower left branch of the limit cycle, synchrony was always the final solution.

As with traveling waves, rotating waves in 2-D lattices need a highly specific positioning on the limit cycle to occur and are not observed frequently when initial conditions are randomly generated. This is explained by the fact that a rotating wave needs at least two or more oscillators or groups of oscillators to be active at any given time. As the time spent on the right branch decreases, the temporal ordering of the oscillators must become more and more precise, and the number of oscillators required for a rotating wave increases. The frequency with which rotating waves are observed decreases as $B_{r}$ decreases. We refer the reader to [5] for a more complete description of this behavior. Note that traveling or rotating waves were not observed in 1-D and 2-D locally coupled networks of spike oscillators [7].

In Fig. 14(b), we display $\left\langle T_{S}\right\rangle / \tau_{S}$ as a function of $\log _{10}(2 L-1)$ for square networks with $L$ varying from 2 to 500 with $B_{r}=0.33$. Even though synchrony is always achieved, there is no obvious scaling behavior. The curve is nonmonotonic and nonintuitive. We have no theoretical understanding of how this curve arises. Note the exceptionally short synchronization times.

\section{Discussion}

Synchronization phenomena are widely observed in nature, and are the subject of intense research. Thus far, the study of coupled oscillator systems has revealed a variety of behaviors, including synchrony [18], [31], phase transitions [24], [41], partial synchrony [10], antiphase solutions [23], traveling waves [14], [40], cluster formation and dissipation [12], and self-organized criticality [4]. The complexity of oscillator systems arises in part from the variety of their components, and determining factors include the nature of the oscillator, the form of the interaction (as well as its amplitude, time delay, spatial extent, and homogeneity), the distribution of intrinsic frequencies, external driving forces, and noise.

We numerically examined how the rate of synchrony changes in networks of relaxation oscillators as we changed the nature of the oscillation. Four different classes of relaxation oscillator were studied with a Heaviside coupling, and three different synchronization rates were observed.$\left\langle T_{S}\right\rangle \sim \log (n)$ was observed for relaxation oscillators in the spiking regime for chains of length $n$ and $\left\langle T_{S}\right\rangle \sim \log (L)$ for 2-D square networks of length $L .\left\langle T_{S}\right\rangle \sim n^{p}$ was observed for relaxation oscillators in the singular limit. $\left\langle T_{S}\right\rangle \sim n$ was observed for relaxation oscillators and relaxation oscillators in the sinusoidal regime. These results 
indicate that the kind of the oscillation changes the rate of synchronization.

We note that for small values of $p$, the function $n^{p}$ appears as a straight line on a semilog plot. Therefore, $\left\langle T_{S}\right\rangle \sim n^{p}$ can be hard to distinguish from logarithmic scaling $\left\langle T_{S}\right\rangle \sim \log (n)$ for even relatively large chain lengths. From our data we cannot exactly determine whether or not the scaling relation is logarithmic or a power law with small $p$.

For relaxation oscillator chains with $\varepsilon>0$, when the coupling changed from a nearly discontinuous step function to a smooth sigmoid, the synchronization rate changed from $\left\langle T_{S}\right\rangle \sim$ $n$ to $\left\langle T_{S}\right\rangle \sim n^{2}$. These results indicate that the form of the coupling modifies the synchronization rate.

All of our numerical results were performed using finite systems. Although the aforementioned trends are apparent in our data, we do not know with certainty that they accurately reflect the asymptotic behavior of the systems studied.

Somers and Kopell [39] emphasized the role of relaxation oscillations in obtaining quick synchrony and suggested that "fast threshold modulation" (FTM) [39], [40] is responsible for the linear scaling relationship. Though they used a Morris-Lecar oscillator with a sigmoid interaction term multiplied by the voltage-like variable, the same basic dynamics of FTM are observed with Terman-Wang oscillators in the relaxation regime. For Terman-Wang oscillators in the sinusoidal regime, the dynamics of FTM are not observed and the linear scaling relation persists, thus, suggesting that an additional mechanism exists that causes the linear scaling relationship.

We also note that the coupling term is a nontrivial function of the fast variable. When the position of the oscillator changes rapidly, the interaction term exhibits rates of change accordingly. In this sense, the nature of the relaxation oscillation causes the interaction term to become step-like. Our data suggest that as $\varepsilon$ becomes smaller the system exhibits synchronization times that are shorter than $\left\langle T_{S}\right\rangle \sim n^{2}$, but we do not have a good estimate for what value of $\varepsilon$ is required for a relaxation oscillator network of a given size and interaction to exhibit $\left\langle T_{S}\right\rangle \sim n$.

We conjecture that a discontinuous interaction has better properties of synchronization than smooth interactions in many oscillator systems. This is consistent with Daido's argument that a step-like coupling "embodies a limit of strong coupling because it yields finite synchronizing force even from an infinitesimal phase difference" [10] (see also [21]). We note that another benefit of a step-like coupling is that synchronization occurs quicker.

Our conjecture may have practical applications. Resonance tunneling diodes [8], [50] oscillate at megahertz and higher frequencies. However, the output current of these devices is very small and there is a need to couple many diodes together to create devices that yield high-output currents at these frequencies. Similarly, phase synchrony of Josephson arrays is desirable [15]. At the moment, it is unknown how to quickly synchronize the outputs of locally coupled arrays of these devices. Even a partial understanding of how to achieve quick synchrony in the presence of noise and disorder would be valuable. Step-like, or pulsatile interactions may also be useful in synchronization of chaotic systems (see [26], [42] for example).
While emphasizing the flexibility of relaxation oscillators, we have only discussed a limited set of relaxation oscillator systems and our results are focussed almost entirely on synchronization. Networks of relaxation oscillators can exhibit a variety of behaviors. With inhibitory coupling, our experiments showed that chains of oscillators quickly attained an entrained state. For relaxation oscillators with an intermediate time scale on the active phase of the limit cycle, slower than the transition time scale, but faster than the slow time scale, almost synchronous solutions arise, and desynchronous solutions exist also [3]. With time-delay coupling, chains of Terman-Wang relaxation oscillators with Heaviside coupling exhibit a rapid approach to a loosely synchronous solution [6], although antiphase solutions exist dependent on the coupling strength and the time delay. For other relaxation oscillators, the synchronous solution can be stable even in the presence of time delays [13]. For a pair of piece-wise linear relaxation oscillators in the singular limit, in-phase and antiphase solutions arise dependent on the initial conditions, the rate of decay of the interaction, and the coupling strength [37] (also see [9]). With diffusive coupling, lattices of relaxation oscillators can synchronize but the coupling strength must be large enough [1]. In a chain of relaxation oscillators with different intrinsic frequencies and diffusive coupling, synchrony is attained, but exhibits extremely long transients even in the presence of large coupling [29]. These behaviors reveal the complexity and subtlety of the systems involved.

\section{REFERENCES}

[1] V. S. Afraimovich, S.-N. Snor, and J. K. Hale, "Synchronization in lattices of coupled oscillators," Physica D, vol. 103, pp. 442-451, 1997.

[2] C. M. Bender and S. A. Orszag, Advanced Mathematical Methods for Scientists and Engineers. New York: McGraw-Hill, 1978.

[3] A. Bose, N. Kopell, and D. Terman, "Almost-synchronous solutions for mutually coupled excitatory neurons," Physica D, vol. 140, pp. 69-94, 2000.

[4] S. Bottani and B. Delamotte, "Self-organized-criticality and synchronization in pulse coupled relaxation oscillator systems: The Olami, feder and christensen and the feder and feder model," Physica D, vol. 103, pp. 430-441, 1997.

[5] S. R. Campbell, "Synchrony and desynchrony in neural oscillators," Ph.D. dissertation, Dept. Phys., The Ohio State Univ., Columbus, OH, 1997.

[6] S. R. Campbell and D. L. Wang, "Relaxation oscillators with time delay coupling," Physica D, vol. 111, pp. 151-178, 1998.

[7] S. R. Campbell, D. L. Wang, and C. Jayaprakash, "Synchrony and desynchrony in integrate-and-fire neurons," Neural Computat., vol. 7, pp. 1595-1619, 1999.

[8] C. L. Chen, R. H. Mathews, L. J. Mahoney, S. D. Calawa, J. P. Sage, K. M. Molvar, C. D. Parker, P. A. Maki, and T. C. L. G. Sollner, "Resonant-tunneling-diode relaxation oscillator," Solid State Elect., vol. 44, pp. 1853-1856, 2000.

[9] S. Coombes, "Phase-locking in networks of pulse-coupled McKean relaxation oscillators," Physica D, vol. 282, pp. 1-16, 2001.

[10] H. Daido, "A solvable model of coupled limit-cycle oscillators exhibiting partial perfect synchrony and novel frequency spectra," Physica $D$, vol. 69, pp. 394-403, 1993.

[11] G. B. Ermentrout and N. Kopell, "Multiple pulse interactions and averaging in coupled neural oscillators," J. Math. Biol., vol. 29, pp. 195-217, 1991.

[12] U. Ernst, K. Pawelzik, and T. Geisel, "Synchronization induced by temporal delays in pulse-coupled oscillators," Phys. Rev. Lett., vol. 74, pp. 1570-1573, 1995.

[13] J. J. Fox, C. Jayaprakash, D. L. Wang, and S. R. Campbell, "Synchronization in relaxation oscillator networks with conduction delays," Neural Computat., vol. 13, pp. 1003-1021, 2001.

[14] P. Goel and B. Ermentrout, "Synchrony, stability, and firing patterns in pulse-coupled oscillators," Physica D, vol. 163, pp. 191-216, 2002. 
[15] G. Filatrella and N. F. Pederson, "The mechanism of synchronization of Josephson arrays coupled to a cavity," Physica C, vol. 372-376, pp. 11-13, 2002.

[16] R. Fitzhugh, "Impulses and physiological states in theoretical models of nerve membrane," Biophys. J., vol. 1, pp. 445-466, 1961.

[17] J. Grasman and M. J. W. Jansen, "Mutually synchronized relaxation oscillators as prototypes of oscillating systems in biology," J. Math. Biol., vol. 7, pp. 171-197, 1979.

[18] X. Guardiola, A. Diaz-Guilera, M. Llas, and C. J. Perez, "Synchronization, diversity, and topology of networks of integrate and fire oscillators," Phys. Rev. E, vol. 62, no. 4, pp. 5565-5570, 2000.

[19] A. L. Hodgkin and A. F. Huxley, "A quantitative description of membrane current and its application to conduction and excitation in nerve," J. Physiol., vol. 117, pp. 500-544, 1952.

[20] J. J. Hopfield and A. V. M. Herz, "Rapid local synchronization of action potentials: Toward computation with coupled integrate-and-fire oscillator neurons," Proc. Nat. Acad. Sci. USA, vol. 92, pp. 6655-6662, 1995.

[21] E. M. Izhikevich, "Phase equations for relaxation oscillators," SIAM J. Appl. Math., vol. 60, no. 5, pp. 1789-1805, 2000.

[22] N. Kopell and G. B. Ermentrout, "Symmetry and phaselocking in chains of weakly coupled oscillators," Comm. Pure Appl. Math., vol. 39, pp. 623-660, 1986.

[23] N. Kopell and D. Somers, "Anti-phase solutions in relaxation oscillators coupled through excitatory interactions," J. Math. Biol., vol. 33, pp. 261-280, 1995

[24] Y. Kuramoto, Chemical Oscillators, Waves and Turbulence. Berlin, Germany: Springer-Verlag, 1984.

[25] P. S. Linsay and D. L. Wang, "Fast numerical integration of relaxation oscillator networks based on singular limit solutions," IEEE Trans. Neural Networks, vol. 9, pp. 523-532, May 1998.

[26] Z. Liu, Y.-C. Lai, and F. C. Hoppensteadt, "Phase clustering and transition to phase synchronization in a large number of coupled nonlinear oscillators," Phys. Rev. E, vol. 63, 2001. 055201.

[27] K. MacLeod and G. Laurent, "Distinct mechanisms for synchronization and temporal patterning of odor-encoding neural assemblies," Science, vol. 274, pp. 1868-1871, 1996.

[28] E. Mayeri, "A relaxation oscillator description of the burst-generating mechanism in the cardiac ganglion of the lobster, homarus americanus," J. Gen. Physiol., vol. 62, pp. 473-488, 1973.

[29] G. S. Medvedev and N. Kopell, "Synchronization and transient dynamics in the chains of electrically coupled fitzhugh-nagumo oscillators," SIAM J. Appl. Math., vol. 61, no. 2, pp. 1762-1801, 2001.

[30] C. Morris and H. Lecar, "Voltage oscillations in the barnacle giant muscle fiber," Biophys. J., vol. 35, pp. 193-213, 1981.

[31] R. E. Mirollo and S. H. Strogatz, "Synchronization of pulse-coupled biological oscillators," SIAM J. Appl. Math., vol. 50, pp. 1645-1662, 1990.

[32] J. Nagumo, S. Arimoto, and S. Yoshizawa, "An active pulse transmission line simulating nerve axon," Proc. IEEE, vol. 50, pp. 2061-2070, 1962.

[33] S. Neuenschwander and F. J. Varela, "Visually triggered neuronal oscillations in the pigeon: An autocorrelation study of tectal activity," Eur. J. Neurosci., vol. 5, no. 7, pp. 870-881, 1993.

[34] R. E. Plant, "A fitzhugh differential-difference equations modeling recurrent neural feedback," SIAM J. Appl. Math., vol. 40, pp. 150-162, 1981.

[35] J. C. Prechtl, "Visual motion induces synchronous oscillations in turtle visual cortex," Proc. Natl Acad. Sci., vol. 91, pp. 12467-12471, 1994.

[36] W. H. Press, S. A. Teukolsky, W. T. Vetterling, and B. P. Flannery, Numerical Recipes in C: The Art of Scientific Computing, 2nd ed. Cambridge, U.K.: Cambridge Univ. Press, 1992.

[37] Y. D. Sato and M. Shiino, "Spiking neuron models with excitatory or inhibitory synaptic couplings and synchronization phenomena," Phys. Rev. E, vol. 66, no. 4, 2002. 041903.

[38] W. Singer and C. M. Gray, "Visual feature integration and the temporal correlation hypothesis," Ann. Rev. Neurosci., vol. 18, pp. 555-586, 1995.

[39] D. Somers and N. Kopell, "Rapid synchronization through fast threshold modulation," Biol. Cybern., vol. 68, pp. 393-407, 1993.

[40] _ - "Waves and synchrony in networks of oscillators of relaxation and nonrelaxation type," Physica D, vol. 89, pp. 169-183, 1995.
[41] S. H. Strogatz, "From kuramoto to crawford: Exploring the onset of synchronization in populations of coupled oscillators," Physica D, vol. 143, pp. 1-20, 2000

[42] J. Teramae and Y. Kuramoto, "Strong desynchronizing effects of weak noise in globally coupled systems," Phys. Rev. E, vol. 63, no. 3, 2001. 036210 .

[43] D. Terman and D. L. Wang, "Global competition and local cooperation in a network of neural oscillators," Physica D, vol. 81, pp. 148-176, 1995.

[44] B. van der Pol, “On Relaxation oscillations," Phil. Mag., vol. 2, no. 11, pp. 978-992, 1926.

[45] B. P. van der and J. M. van der, "The Heartbeat Considered as a Relaxation Oscillation, and an Electrical Model of the Heart," Phil. Mag., ser. 7, pt. X, vol. 6, pp. 763-775, 1928.

[46] D. L. Wang, "Emergent synchrony in locally coupled neural oscillators," IEEE Trans. Neural Networks, vol. 6, pp. 941-948, July 1995.

[47] — "Relaxation Oscillators and Networks," in Wiley Encyclopedia of Electrical and Electronics Engineering, J. Webster, Ed. New York: Wiley, 1999, vol. 18, pp. 396-405.

[48] H. R. Wilson and J. D. Cowan, "Excitatory and inhibitory interactions in localized populations of model neurons," Biophys. J., vol. 12, pp. 1-24, 1972.

[49] A. T. Winfree, The Geometry of Biological Time. New York: Scientific Book, 1980

[50] J. F. Young, B. M. Wood, H. C. Liu, M. Buchanan, D. Landheer, A. J. SpringThorpe, and P. Mandeville, "Effect of circuit oscillations on the dc current-voltage characteristics of double barrier resonant tunneling devices," Appl. Phys. Lett., vol. 52, pp. 1398-1401, 1988.

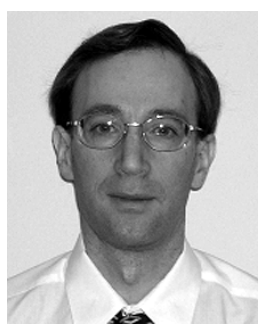

Shannon R. Campbell received the B.S. degree in physics and mathematics from the University of California, Davis, in 1990 and the Ph.D. degree in physics from The Ohio State Univerity, Columbus, in 1997.

From 1997 to 2004, he held various positions in industry and at the National Institutes of Health, Bethesda, MD, working in image processing and pattern recognition applications. He is currently supported by SBIR Grants and his research interests are in pattern recognition, image processing, neurodynamics, and human visual perception.

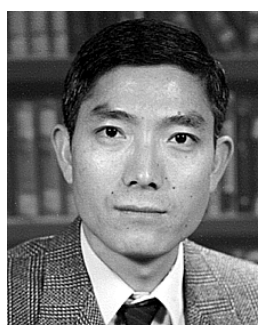

DeLiang Wang (M'90-F'04) received the B.S. and M.S. degrees in computer science from Peking (Beijing) University, Beijing, China, in 1983 and 1986, respectively, and the Ph.D. degree from the University of Southern California, Los Angeles, in 1991.

From 1986 to 1987, he was with the Institute of Computing Technology, Academia Sinica, Beijing, China. Since 1991, he has been with the Department of Computer and Information Science and the Center for Cognitive Science, The Ohio State University, Columbus, where he is currently a Professor. From 1998 to 1999, he was a Visiting Scholar in the Department of Psychology, Harvard University, Cambridge, MA. His research interests include machine perception and neurodynamics.

Dr. Wang currently chairs the IEEE Neural Networks Society Neural Networks Technical Committee, is a member of the Governing Board of the International Neural Network Society and the IEEE Signal Processing Society Machine Learning for Signal Processing Technical Committee. He is a recipient of the U.S. Office of Naval Research Young Investigator Award.

Ciriyam Jayaprakash, photograph and biography not available at the time of publication. 